\title{
WOLF-RAYET STARS IN GALAXIES
}

\section{AND THE ROLE OF BINARIES AND HYDRODYNAMICS}

\author{
ANDRÉ MAEDER \\ Geneva Observatory, CH-1290 Sauverny, Switzerland
}

\begin{abstract}
WR stars obey $M-L-\dot{M}-T_{\text {ef } f}-R$-chemistry relations, which are in general model independent (with the exception of chemistry). The processes of WR formation (stellar winds, hydrodynamical mixing, binary mass transfer etc.) influence, however, the domain of parameters occupied by WR stars. We specifically examine the distribution of luminosities and $\mathrm{H}$-contents of WN stars, which both support heavy mass loss rates, or possibly mixing, in the main sequence and WNL phases. Detailed studies of the number ratios WR/O, WC/WN, WC/WR, etc. are made for galaxies at various metallicities $Z$ which exhibit extremely different WR populations. Good agreement of models and observations is found. It is also shown that the data are better explained if a certain fraction $\phi$ (less than 10\%) of the O-stars become WR stars, preferentially of type WNE as a result of Roche lobe overflow (RLOF) in binaries. This result necessarily implies that the fraction of WR stars, owing their existence to RLOF, is variable with $Z$, being nearly $100 \%$ at low $Z$ and much smaller at high $Z$. We also identify several hydrodynamical developments physically required in stellar models. Among them, we collect the available observational and theoretical arguments supporting an important role of mixing in massive star evolution.
\end{abstract}

Key words: stars: Wolf-Rayet - close binaries - mass-loss - evolution - hydrodynamics

\section{Introduction: the importance of WR stars}

To some of our colleagues, the study of WR stars looks like a curious astronomical hobby. However, one must emphasize that WR stars are the only stars which may be identified and counted in distant galaxies, thus providing direct tracers of star formation. Also, WR stars are the last visible stage in evolution before supernova explosions. Thus, we must understand the remarkable properties of WR stars in galaxies of various metallicities $Z$, in order to properly describe and modelise massive star evolution, nucleosynthesis and the chemical evolution of galaxies. On the whole, WR stars are a key piece in the understanding of the distant galaxies and of their chemical evolution.

\section{Basic WR properties, chemistry and observational comparisons}

WR stars are essentially bare cores. In the WNL stage, with generally $\mathrm{H}$ present, models show that the luminosities are related to the initial stellar masses ( $c f$. cases of 40 and $60 \mathrm{M}_{\odot}$ in Fig. 1), because these determine the size of the He-cores. However, in case of extreme mass loss leading to an entry in the WNL stage (as judged from surface composition) during the 
H-burning phase, the He-core heavily decreases and leads to very low WNL luminosities ( $c f$. case of $120 \mathrm{M}_{\odot}$ in Fig. 1).

In the WNE and WC stages, the internal composition and structure are rather simple. Therefore, models predict $M-L-\dot{M}-T_{\text {eff }}-R$-composition relations (Schaerer \& Maeder, 1992). For example, the $L-T_{\text {eff }}$-relations for WNE and WC stars are illustrated in Fig. 1. The $M-L$ relation is (Maeder 1983; Langer 1989a; Beech \& Mitalas 1992; Schaerer \& Maeder 1992):

$$
\log \frac{L}{\mathrm{~L}_{\odot}}=3.032+2.695 \log \frac{M}{\mathrm{M}_{\odot}}-0.461\left(\log \frac{M}{\mathrm{M}_{\odot}}\right)^{2}
$$

For $M>10 \mathrm{M}_{\odot}$, a linear fit is sufficient

$$
\log \frac{L}{\mathrm{~L}_{\odot}}=3.495+1.727 \log \frac{M}{\mathrm{M}_{\odot}}
$$

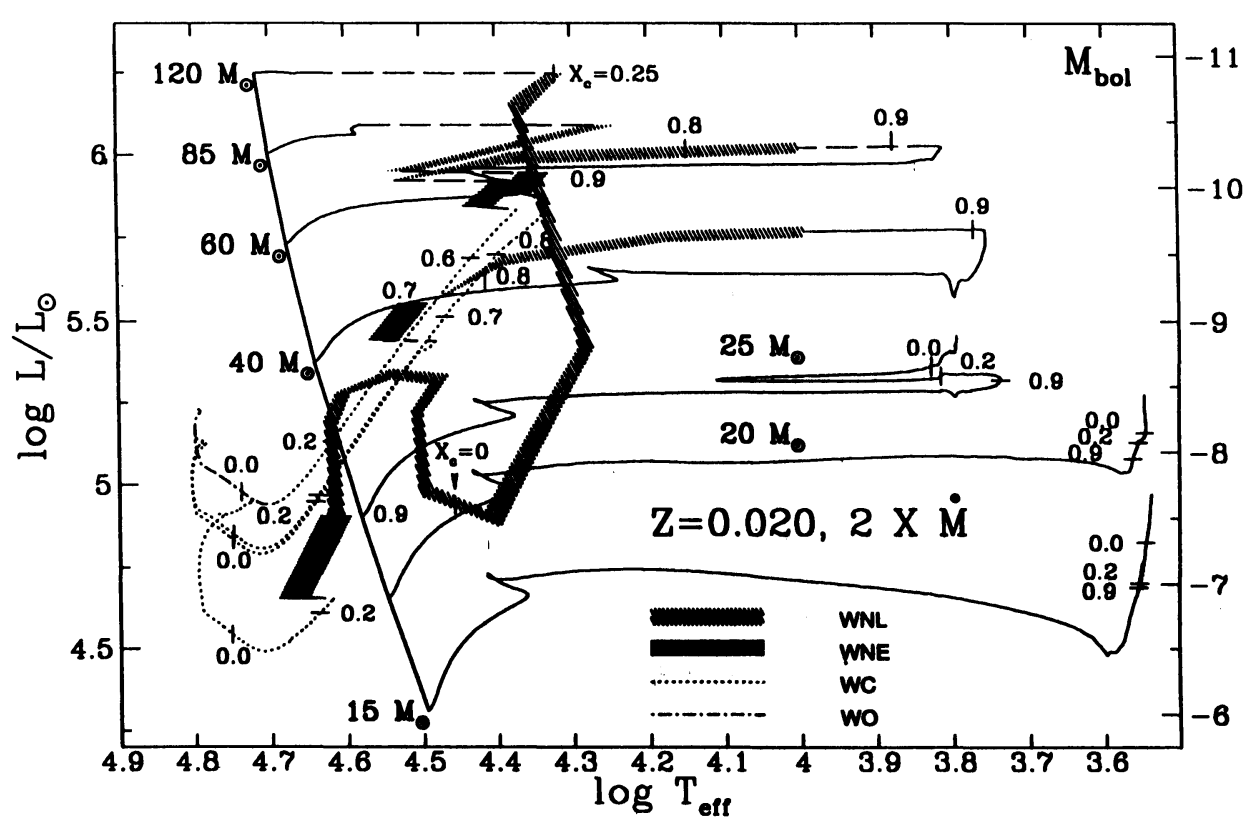

Fig. 1. Evolutionary tracks for $Z=0.02$ and increased mass loss rates before the WNE stages. The central helium content are indicated along the tracks. The various WR subgroups are shown, (Meynet et al. , 1994). 
In first approximation, we can say that the $M-L-\dot{M}-T_{\text {eff }}-R$ are independent of the possible modes of WR formation: stellar winds, hydrodynamical assumptions, mixing, RLOF in binaries, etc.. However, the range of parameters occupied by WR stars along these various relations may be quite different according to the process of formation and their importance. In comparison with observations, it is thus important to examine the domain occupied by WR stars.

Let us now turn towards observations. The mass-luminosity relation is well confirmed observationally (Smith \& Maeder 1989; Smith et al. 1994). WR stars show some alignments in the HR diagram (Hamann et al. 1993; Langer et al. 1994). We notice that the models of Fig. 1, which include an appropriate correction for the optically thick winds, correctly predict the location of WR stars in the HR diagram. It will be most interesting to have the results of the unified stellar models ( $c f$. Schaerer, these proceedings). In Table I, the average luminosities, the standard deviation around the average and the minimum luminosities of WN stars by Hamann et al. (1993) are compared with model predictions for an IMF with Salpeter's slope. We point out that models with enhanced mass loss by Meynet et al. (1994), with metallicities between $Z=0.02$ and 0.04 well reproduce the observed luminosities.

TABLE I

Comparison of observed and theoretical luminosities of WN stars.

\begin{tabular}{lcccccccccc}
\hline WR & \multicolumn{3}{c}{ observation } & \multicolumn{3}{c}{ theory } & \multicolumn{3}{c}{ theory } \\
subtype & $\langle\log L>$ & $\sigma$ & $\log L_{\text {min }}$ & $\langle\log L>$ & $\sigma$ & $\log L_{\text {min }}$ & $\langle\log L>$ & $\sigma$ & $\log L_{\text {min }}$ \\
\hline & & & & \multicolumn{4}{c}{$Z=0.020$} \\
WN & 5.3 & 0.4 & 4.4 & 5.5 & 0.4 & 4.7 & 4.6 & 0.7 & 3.3 \\
WNL & 5.4 & 0.4 & 4.4 & 5.6 & 0.4 & 4.9 & 4.6 & 0.9 & 3.3 \\
WNE & 5.1 & 0.3 & 4.6 & 5.2 & 0.4 & 4.7 & 4.6 & 0.5 & 4.0 \\
\hline
\end{tabular}

Detailed comparisons between observed and theoretical abundances have been performed by Willis (1991). For WN stars, the observed abundance ratios correspond to equilibrium values of the CNO cycle. Due to equilibrium, these ratios are independent of model assumptions and thus the good observed agreement yields the important conclusion that our knowledge of the main CNO reactions is correct. We emphasize that WN stars are the only stars where pure equilibrium ratios of the CNO cycle can be observed, both in the presence of hydrogen (WNL) and in absence of it (WNE).

Recently, Langer et al. (1994) have proposed new models leading to WR stars. They examine the plot of the surface hydrogen mass fraction $v s$. the 
luminosities of WN stars. Figure 2 clearly shows that a lot of WNL stars with low $\mathrm{H}$-content are observed. However, the models they give spend a negligible time $\left(3 \cdot 10^{4} \mathrm{yr}\right)$ in this low $\mathrm{H}$ domain. Fig. 2 shows the same data with the superposition of the recent models by Meynet et al. (1994) with enhanced mass loss rates in pre-WNE stages. The agreement is much better, especially for models with $Z=0.04$. The question of WN stars with low luminosities and $\mathrm{H}$-content around 0.2 remains open.

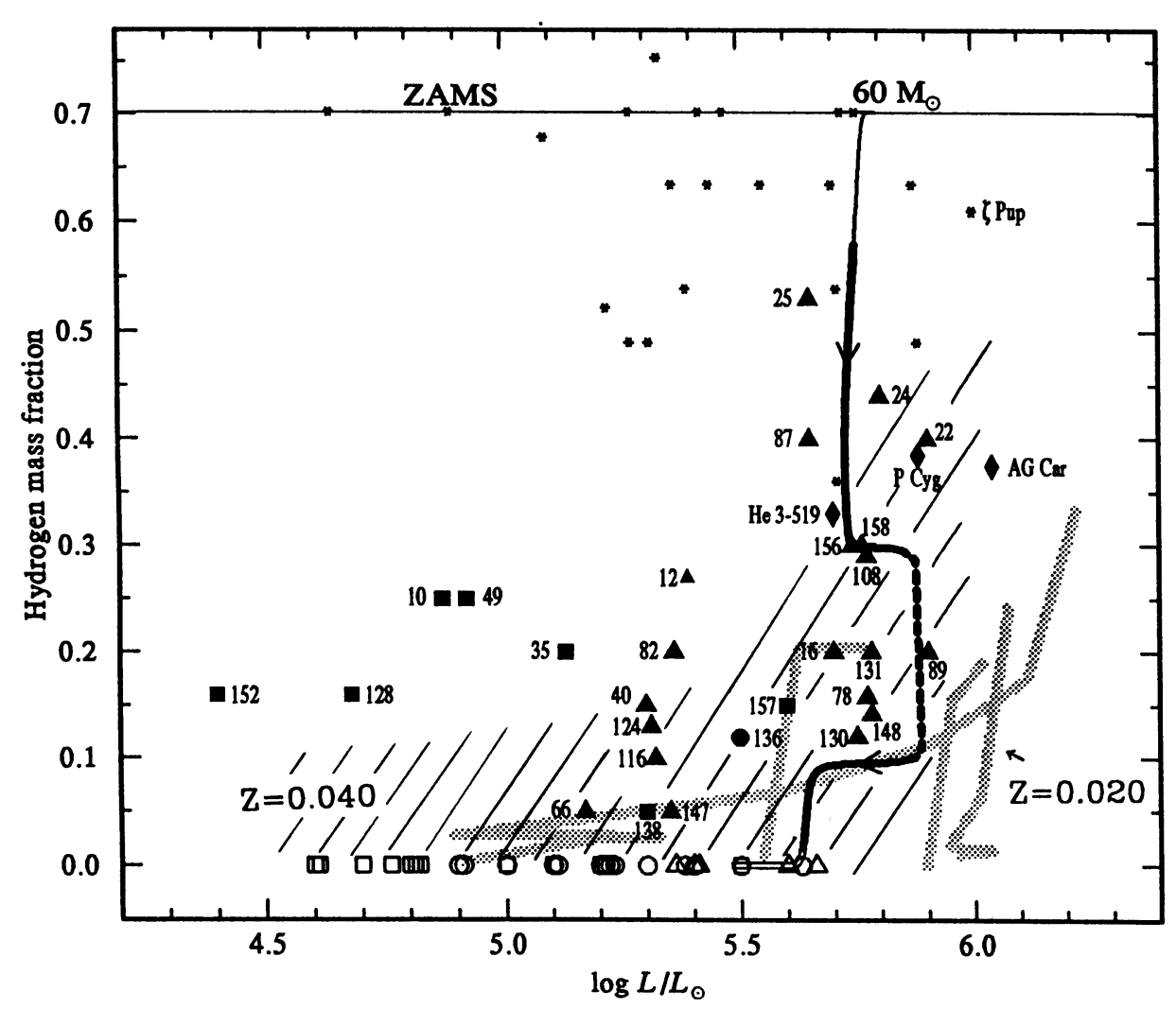

Fig. 2. H-abundance vs. luminosity for WN stars (Langer et al. , 1994). Open symbols refer to object without $\mathrm{H}$ signatures. Triangles, squares and circles refer to WNL, WNE (weak lines), WNE (strong lines). The location of models by Meynet et al. (1994) is shown, with shaded are for the zone of maximum expectations.

In WC stars, the products of partial He-burning are present. The abundance ratios are not equilibrium values and are thus very dependent on model assumptions. Comparisons generally show a good agreement (Willis, 1991). Each WC subtype corresponds to different values of the $(\mathrm{C}+\mathrm{O}) / \mathrm{He}$ ratio (Smith \& Maeder, 1991). This calibration is indicated on Fig. 3, which also shows the model predictions for the $(\mathrm{C}+\mathrm{O}) / \mathrm{He}$ ratios as a function of initial 
mass and $Z$. Remarkably, this Figure reproduces the main properties of WC stars in galaxies ( $c f$. Smith \& Maeder 1991):

- Late WC stars (WCL) are found at high $Z$ (cf. van der Hucht et al. 1988; Conti \& Vacca 1990).

- At a given $Z$, WCL are brighter than early WC stars (WCE).

- Stars of a given WC subtype are brighter in galaxies with low $Z$.

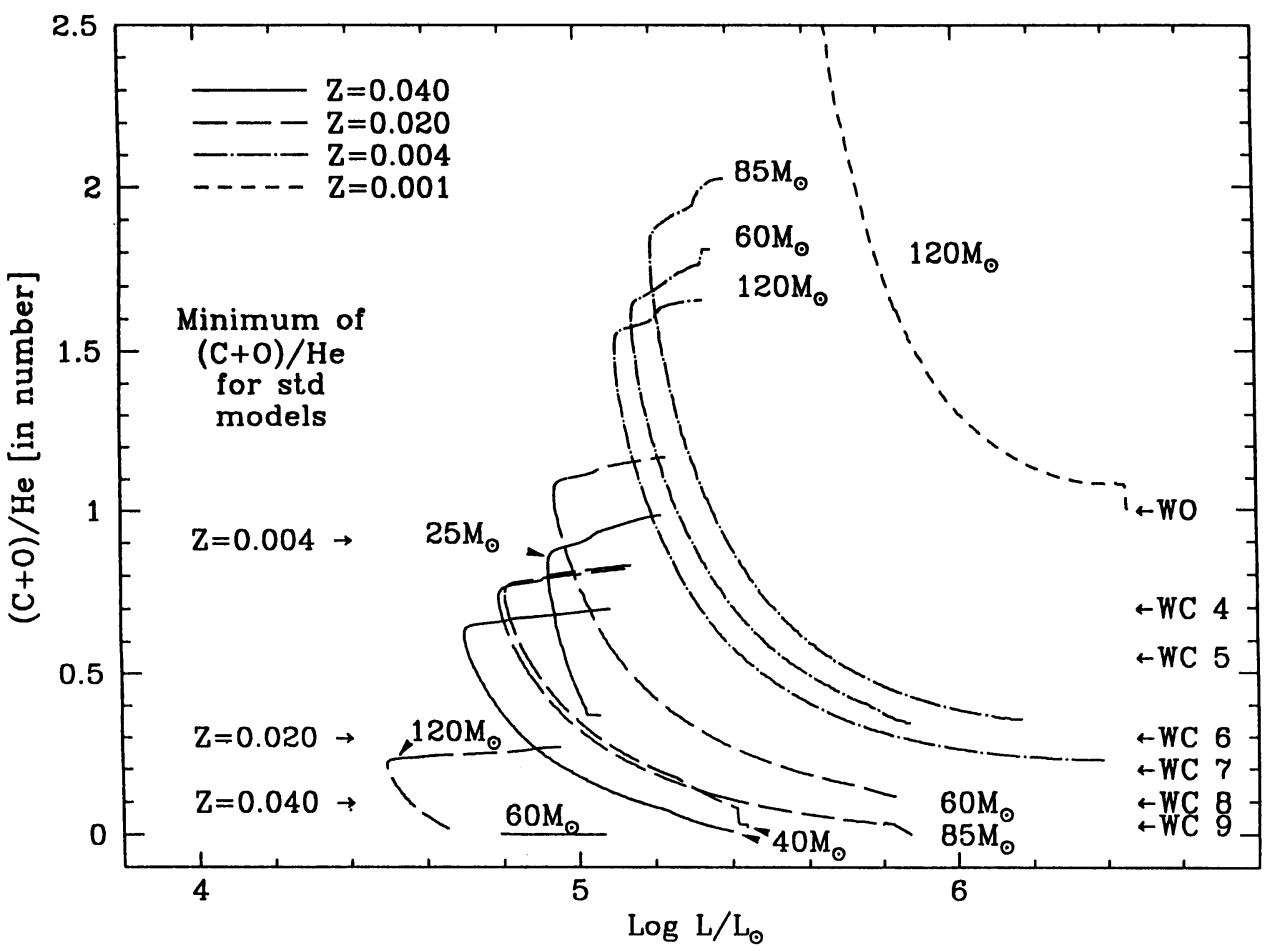

Fig. 3. The $(\mathrm{C}+\mathrm{O}) / \mathrm{He}$ ratio vs. luminosities for WC stars. The observed values for each subtypes are indicated on the right (Smith \& Maeder, 1991). The model predictions by Maeder \& Meynet, (1994) are shown.

\section{WR stars in galaxies and the role of binaries}

The striking changes of the relative frequencies of WR stars in galaxies is a major property which is to be explained. The WR/O and WC/WN ratios show a general increase with the metallicity $Z$ of the parent galaxy (see Fig. 4 and 5). Data have been collected recently by Maeder \& Conti (1994) (see also Maeder 1991; Armandoff \& Massey 1991). Despite the many claims that the changes of WR populations are due to effect of star formation and 
IMF, the main effect is the metallicity $Z$, which through the stellar winds influences stellar evolution, and thus the WR lifetimes.

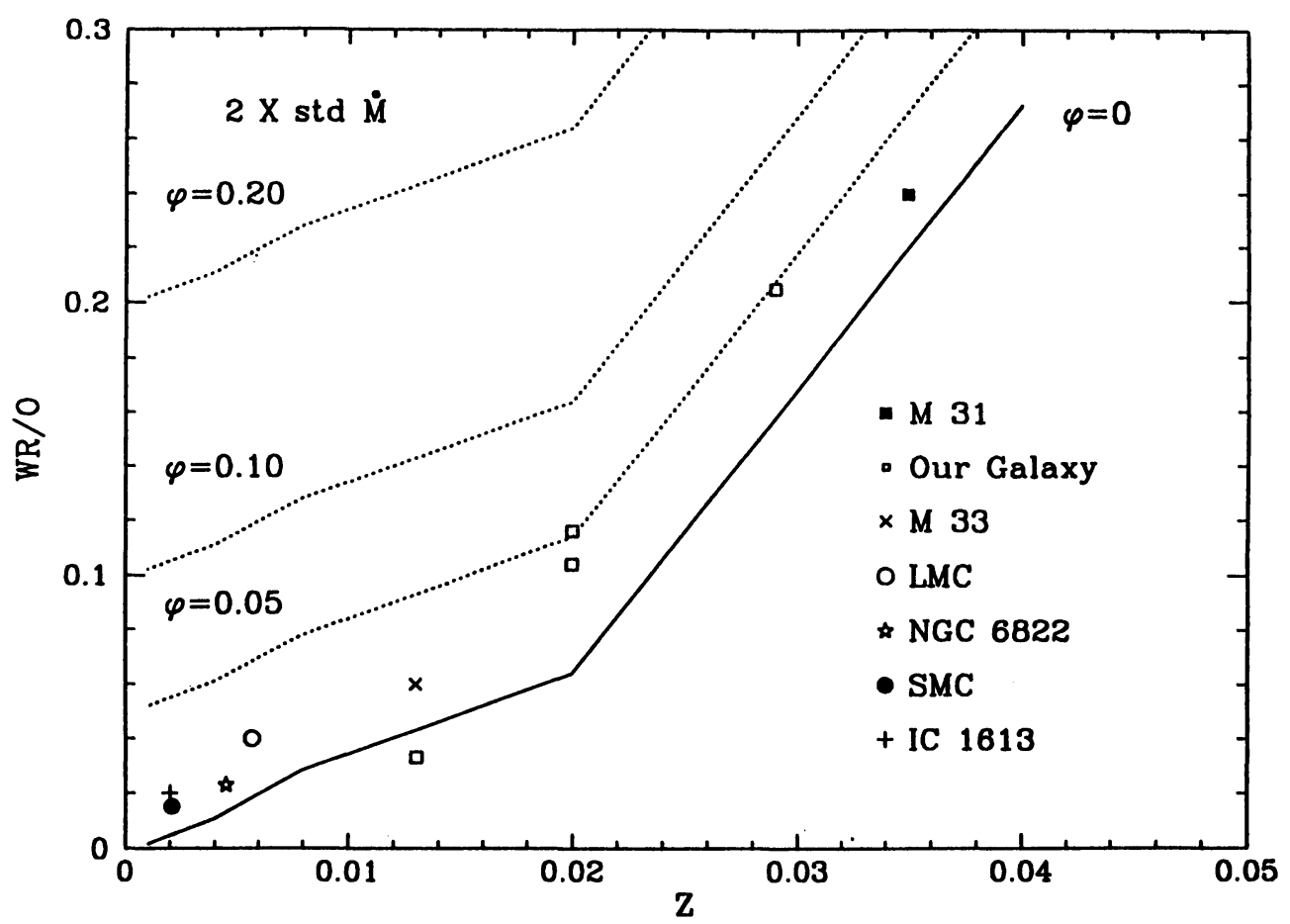

Fig. 4. The observed and predicted number ratio WR/O. Data collected by Maeder \& Conti (1994). Model predictions by Maeder \& Meynet (1994) with Salpeter's IMF slope and enhanced mass loss rates are shown for different values of $\phi$, the fraction of $O$-stars undergoing RLOF and being WR stars.

The duration of the WNL phase depends on the internal H-profile. For important mass loss during the main sequence phase (or mixing !), there is a large zone of varying $\mathrm{H}$ content and this gives rise to long WNL phases. This explains, as shown by Maeder \& Meynet (1994) the strikingly low value of the number ratio WC/WN in M31 found by Armandoff \& Massey (1991): at high Z, due to heavy mass loss, the WNL phase has already been entered during the H-burning stage. Oppositely, the duration of the WNE phase is longer for lower initial masses. This duration is proportional to the mass between the $\mathrm{He}-$-core and the $\mathrm{H}$-shell and inversely proportional to the mass loss rates which in this phase goes like $M^{2.5}$ (Langer, 1989b). Thus, on the whole, the duration of the WNE phase increases with decreasing initial masses.

Figs. 4 and 5 show the comparison between models and observations for the number ratios $\mathrm{WR} / \mathrm{O}$ and $\mathrm{WC} / \mathrm{WR}$. The observed trends are remarkably well reproduced. The Geneva models also explain well the distributions 
of WC subtypes in galaxies (cf. Smith \& Maeder 1991): in particular the presence of WC8 and WC9 in inner galactic regions, of only WC4 and WC5 in the LMC and the higher proportion of WO stars in low $Z$ regions. We also have to account for the WR stars formed by RLOF in binaries (de Loore 1982; de Greve 1991; Vanbeveren 1991; Vanbeveren \& de Loore 1993). Let us call $\phi$ the fraction of $\mathrm{O}$-stars being WR stars as a result of RLOF, and let us assume that $\phi$ is independent on $Z$. Models by de Loore \& Vanveberen (1994) show that the RLOF preferentially leads to WNE stars. Also, the example of the SMC, where the winds are likely to be weak, supports this view. Out of $9 \mathrm{WR}$ stars, 8 are WNE stars likely to result mainly from binary mass transfer (de Greve et al. , 1988).

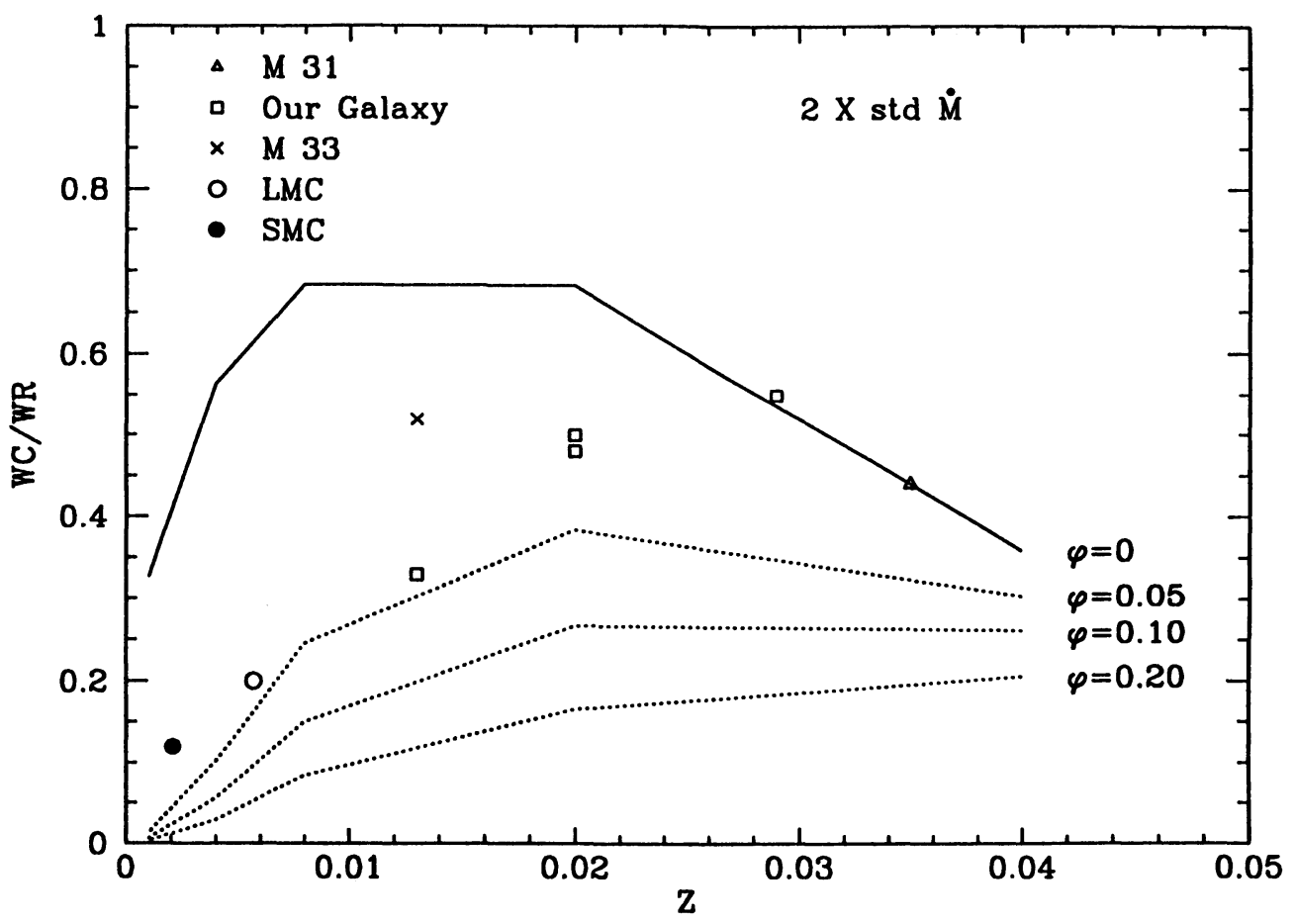

Fig. 5. Same as Fig. 4 for the WC/WR number ratio.

Figs. 4 and 5 suggest that $\phi$ is certainly less than $10 \%$. This is much smaller than the results by Vanbeveren \& de Loore (1993) who estimate that the fraction of all stars undergoing RLOF is about $35 \%$. However, arguments in favour of a low fraction of close binaries undergoing RLOF among massive stars have been provided by Massey (1981) and by Penny et al. (1993). Another argument arises from the fact that the WR/O ratio is 0.017 in the SMC and 0.21 in inner galactic regions. Thus, if the fraction $\phi$ is the same in both areas, it should necessarily be less than $(0.017 / 0.21)=8 \%$, even if 
we assume that all WR stars in the SMC are formed by RLOF.

We also emphasize that the assumption of a constant $\phi$ with $Z$ unavoidably leads to the result that the proportion of WR stars formed by RLOF with respect to all WR stars is highly variable with $Z$. At low $Z$, like in the SMC, it is almost exclusively the binary channel that seems to be able to produce WR stars, while at higher $Z$, stellar winds are very efficient in forming bare cores. The properties of the binary evolution with RLOF have been investigated in several works ( $c f$. Vanbeveren 1991, 1994). In order to better appreciate the various consequences of RLOF, it would be necessary to simulate population models including binary stars with appropriate distributions of mass ratios and separations. A major uncertainty concerns the possibility of mixing induced by tidal effects, these could lead to homogeneous evolution, possibly preventing RLOF.

Finally, we note that the analyses presented here concern galaxies or large areas in galaxies, where constant SFR rates over the last $10^{7} \mathrm{yr}$ can be assumed. This is no longer true in starbursts where age and perhaps IMF effects also intervene.

\section{The future of WR modelling: the role of hydrodynamics}

The hydrostatic models already give far reaching results on WR stars, but clearly further developments are needed. In our opinion, they will mainly concern the model hydrodynamics. At present, we may identify three different kinds of approach to the hydrodynamic problems.

- Instabilities and hydrodynamical model ( $\ddot{r}$ term)

The mutual interactions of evolution, instabilities and mass loss can be particularly important for WR stars. Instabilities can be treated in several ways. For example, Schaller (1992) has coupled a code for non adiabatic, linear and radial oscillations to the evolutionary code. This allows us to identify unstable phases during evolution. In particular, instabilities were found for yellow supergiants and WR stars. The inclusion of the $\ddot{\mathbf{r}}$-term in evolution allows us to consider non-linear effects in instabilities ( $c f$. Meynet 1991; Glatzel et al. 1993). Two interesting results are already there: (1) New instabilities have been found (Glatzel et al. 1993; Kiriakidis et al. 1993); (2) The evolutionary time-scale in non-nuclear phases, such as the crossing of the HR gap occurs on a time-scale shorter by a factor of 2, (cf. Meynet 1991).

- Unified star models

New models are built ( $c f$. Schaerer, these proceedings), which incorporate in a single consistent treatment the stellar interior, the atmosphere and the stellar winds. For the interior, the hydrostatic approximation is used but hydrodynamics needs to be considered in the outer layers. Such models represent an important step forward in stellar modelling, 
as they give the emergent spectrum, with lines included, along the evolutionary tracks.

\section{- Internal hydrodynamics and mixing}

At present, this is the most uncertain and potentially most important problem in star evolution. There are both observational and theoretical reasons suggesting a major role of mixing for massive stars. Among observations, we notice several compelling facts:

- Fast rotators among $\mathrm{OB}$ stars have $\mathrm{He}$ and $\mathrm{N}$ enhancements (Herrero et al. , 1992).

- The existence of ON stars (Walborn, 1988).

- The He and $\mathrm{N}$ enrichments in most $\mathrm{B}$ and $\mathrm{A}$ supergiants (Gies \& Lambert 1992; Venn 1993).

- The large N/C and N/O ratios in SN 1987 A (Fransson et al. , 1989).

- The existence of intermediate WN/WC stars (cf. Conti \& Massey 1989).

On the theoretical side, we may remark that most mixing processes are dissipative and in this case the diffusion coefficient $D$ is somehow related to the viscosity. For example, such a relation occurs for rotational mixing (Zahn, 1992) and for semiconvective mixing (Langer et al. , 1985). In such cases, the characteristic time for mixing is

$$
t_{m i x} \simeq 0.4 R^{2} / \bar{D} \quad \text { where } \quad \bar{D} \text { is the average } D .
$$

For the radiative viscosity

$$
\nu_{r a d}=4 a T^{4} /\left(15 c \kappa \rho^{2}\right) \quad \text { we get } t_{m i x} \simeq 0.1 \frac{c}{4 a}\left(\frac{k}{G \mu m_{H}}\right)^{4} \frac{\kappa}{R e^{\star} M^{2}}
$$

where $R e^{\star}=\bar{D} / \nu$ is of the order of $10^{2}$.

The above expression shows that $t_{m i x}$ decreases much faster than the main sequence life-time $t_{M S}=10^{7.87}\left(M / \mathrm{M}_{\odot}\right)^{-0.72} \mathrm{yr}$, an expression valid in the range of 20 to $85 \mathrm{M}_{\odot}$. These expressions suggest that mixing becomes increasingly significant for larger stellar masses.

Coming back to WR stars, we emphasize that mixing would act in several respects like increased mass loss rates. In particular, mixing during the $\mathrm{H}$ burning phase (Maeder 1987) favours the formation of WR stars and it would lead to the formation of many WR stars, with low $\mathrm{H}$ content, a feature which is required by Fig.2. Mixing in the He-burning phase is required to explain the observed number of intermediate WN/WC stars (cf. Langer 1991). Thus, at the end of this presentation, we may question whether there is room or not for a significant role of internal mixing in the uncertainty range of mass loss rates. The fact that most observations, when interpreted in terms of mass loss, suggest higher mass loss rates in the main sequence phase, could well be a positive indication. 


\section{References}

Armandoff, T.E., Massey, Ph. 1991, AJ 102, 927

Beech, M., Mitalas, R. 1992, $A \mathcal{B} A$ 262, 483

Conti, P.S., Massey, P. 1989, ApJ 337, 251

Conti, P.S., Vacca, W.D. 1990, $A J$ 100, 431

de Greve, J.P. 1991, in: K.A. van der Hucht \& B. Hidayat (eds.), Wolf-Rayet Stars and Interrelations with Other Massive Stars in Galaxies, Proc. IAU Symp. No. 143 (Dordrecht: Kluwer), p. 213

de Greve, J.P.,Hellings, P., van den Heuvel, E.P.J. 1988, $A \& A$ 189, 74

de Loore, C. 1982, in: C.W.H. de Loore \& A.J. Willis (eds.), Wolf-Rayet Stars: Observations, Physics, Evolution, Proc. IAU Symp. No. 99 (Dordrecht: Reidel), p. 343

de Loore, C., Vanbeveren D. 1994, $A \mathscr{B} A$ in press

Fransson, C., Cassatella, A., Gilmozzi, R., Kirshner, R.P., Panagia, N., Sonneborn, G., Wamsteker, W. 1989, ApJ 336, 429

Gies, D.R., Lambert, D.L. 1992, ApJ 387, 673

Glatzel, W., Kiriakidis, M., Fricke, K.J. 1993, MNRAS 262, L7

Hamann, W.-R., Koesterke, L, Wessolowski, U. 1993, $A \& A$ 274, 397

Herrero, A., Kudritzki, R.P., Vilchez, J.M., Kunze, D., Butler, K., Haser, S. 1992, $A \mathscr{E} A$ 261, 209

Kiriakidis, M., Fricke, K.J., Glatzel, W. 1993, MNRAS 264, 50

Langer, N. 1989a, $A \& A$ 210, 93

Langer, N. 1989b, $A \& A 220,135$

Langer, N. 1991, $A \& A$ 248, 531

Langer, N., El Eid, M.F., Fricke, K.J. 1985, $A \& A$ 145, 179

Langer, N., Hamann, W.-R., Lennon, M., Najarro, F., Pauldrach, A.W.A, Puls, J. 1994, $A \mathscr{E} A$ in press

Maeder, A. 1983, $A \mathscr{B} A$ 120, 113

Maeder, A. 1987, $A \cup A$ 178, 159

Maeder, A. 1991, $A \& A$ 242, 93

Maeder, A., Conti, P.S. 1994, Ann. Rev. $A \mathscr{E} A$ in press

Maeder, A., Meynet, G. 1994, $A \& A$ 287, 803

Massey, Ph. 1981, ApJ 246, 153

Meynet, G. 1991, in: C. de Jager \& H. Nieuwenhuijzen (eds.), Instabilities in Evolved Super- and Hypergiants (Amsterdam: North Holland), p. 173

Meynet, G., Maeder, A., Schaller, G., Schaerer, D., Charbonnel, C. 1994, A\&A Suppl. 103,97

Penny, L.R., Gies, D.R., Hartkopf, W.I., Mason, B.D., Turner, N.H. 1993, PASP 105, 588

Schaerer, D., Maeder, A. 1992, A\&A 263, 129

Schaller G. 1992, PhD thesis, University of Geneva

Smith, L.F., Maeder, A. 1989, $A \mathscr{E} A$ 211, 71

Smith, L.F., Maeder, A. 1991, $A \cup A$ 241,77

Smith, L.F., Meynet, G., Mermilliod, J.-C. 1994, $A \& A$ bf 287, 835

Vanbeveren, D. 1991, Space Sci. Rev. 54, 249

Vanbeveren, D. 1994, Space Sci. Rev. 66, 327

Vanbeveren, D., de Loore, C. 1993, in: J.P. Cassinelli \& E.B. Churchwell (eds.), Massive Stars: Their Lives in the Interstellar Medium, ASP. Conf. Ser. 35, 257

van der Hucht, K.A., Hidayat, B., Admiranto, A.G., Supelli, K.R., Doom, C. 1988, $A \& A$ 199,217

Venn, K.A. 1993, ApJ 414, 316

Walborn, N. 1988, in: K. Nomoto (ed.), Atmospheric Diagnostics of Stellar Evolution, Proc. IAU Coll. No. 108 (Berlin: Springer), p. 70

Willis, A.J. 1991, in: G. Michaud \& A. Tutukov (eds.), Evolution of Stars: The Photospheric Abundance Connection, Proc. IAU Symp. No. 145 (Dordrecht: Kluwer), p. 195

Zahn, J.P. 1992, $A \& A$ 265, 115 


\section{DISCUSSION:}

Schmutz: It is likely that WR winds are clumped. This would imply that the mass loss rates are lower by some factor, say 2 or 4 . Would this affect the stellar evolution in that it would be more difficult or even impossible to reach the WC stage?

Maeder: The answer depends whether you are speaking about mass loss rates in the WR stages or before. For WR stages, even if you change the numerical factor in the relation $\dot{\mathrm{M}} \sim \mathrm{M}^{2.5}$, it will not prevent most WR stars to enter the WC stage. However, if you would reduce the mass loss rates in O- and supergiant stages, it would heavily reduce the WR lifetimes, and for the WC phase as well. Simultaneously the predicted luminosities of WR stars would be much too high with respect to the observations by Hamann, for example. This is why I think that either higher mass loss rates, or possibly mixing, in pre-WR stages is very necessary.

Hamann: You have shown that the observed luminosities of the WN stars can be explained with your most recent tracks (Meynet et al. 1994), but this comparison implies that the majority of WN stars (those with lower luminosities) had very massive progenitors with more than 100 $\mathrm{M}_{\mathrm{o}}$ at the ZAMS. Is this consistent with any reasonable initial-mass function?

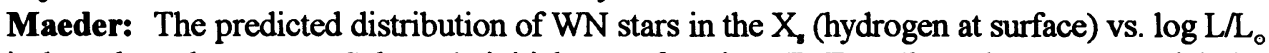
is based on the current Salpeter's initial mass function (IMF). Thus, the recent models by Meynet et al. (1994) will reproduce the observed parameter distribution of WN stars. If one looks carefully to your diagram, one may see that perhaps the only slight problem concerns the existence of WN stars with $\mathrm{X}_{\mathbf{r}}=0.2-0.3$ at low luminosities.

Kaper: 1. Blaauw (1992) suggested that the rapidly rotating OB stars that are runaways (e.g. $\mathrm{S}$ Oph, the most rapidly rotating $\mathrm{O}$ star, $\lambda$ Cep, $\zeta$ Per etc) could have higher abundance and rotation rate due to mass transfer in a binary system that disrupted after the (first) supernova explosion. What is your opinion about this?

2. How far do you have to extrapolate the $M(M)$ relation to obtain mass-loss rates of $10^{-3}-10^{-4}$ $\mathrm{M} \odot / \mathrm{yr}$ ?

Maeder: This is a very reasonable scenario, however to be confirmed it needs a more complete investigation on abundances and rotation in runaway stars. About the second point, the current mass loss formulae predict very high mass loss rates for supergiants, nevertheless specific rates have to be adopted to modelize the LBV phase. 